\title{
New Formulation of the Mulilayer Iterative Method: Application to Coplanar Lines with Thick Conductor
}

\author{
Rafika Mejri, Taoufik Aguili \\ Communication System Laboratory Sys'Com, National Engineering School of Tunis, University of Tunis El Manar, Tunis, Tunisia \\ Email: mejrirafika@yahoo.fr
}

How to cite this paper: Mejri, R. and Aguili, T. (2016) New Formulation of the Mulilayer Iterative Method: Application to Coplanar Lines with Thick Conductor. Journal of Electromagnetic Analysis and Applications, 8, 197218.

http://dx.doi.org/10.4236/jemaa.2016.89019

Received: July 10, 2016

Accepted: September 27, 2016

Published: September 30, 2016

Copyright () 2016 by authors and Scientific Research Publishing Inc. This work is licensed under the Creative Commons Attribution International License (CC BY 4.0).

http://creativecommons.org/licenses/by/4.0/

\begin{abstract}
The skin effect is an electromagnetic phenomenon that makes the current flows only on the surface of the conductors at high frequency. This article is based on the phenomenon to model a structure made in coplanar technology. In reality, these types of structures integrated metal layers of different thickness of copper $(9 \mu \mathrm{m}, 18 \mu \mathrm{m}, 35$ $\mu \mathrm{m}, 70 \mu \mathrm{m})$. The neglect of this parameter introduces errors, sometimes significant, in the numerical calculations. This is why an iterative method (FWCIP) based on the wave concept was restated. Validation of results was carried out by comparison with those calculated by Ansoft HFSS software and Agilent ADS Technology. They show a good matching.
\end{abstract}

\section{Keywords}

Skin Effect, Electromagnetic Phenomenon, Iterative Method, Coplanar Structure, Microwave

\section{Introduction}

In Radio frequency, most devices are made in micro strip technology [1] [2]. This technology became the best known and most used. This is due to its flat nature, ease of manufacturing, low cost, easy integration with circuits in the solid state, good heat dissipation structure that is used as good mechanical support etc. In general and in various research works concerning modeling and study of these structures, most researchers assumed that they possessed metallic conductors without thickness [3] [4]. This simplifying assumption decreases the accuracy of the results of analytical methods used for the characterization of these structures. Several methods were used to characterize the influence of the thickness of the conductor used, such as mode-matching method [5], 
method of lines [6], spectral domain method [7] and conformal mapping method [8]. For the study of structures with flat and thick conductors such as micro strip line in this document, we have taken the first model proposed in [6]. A new formulation of the iterative method FWCIP (Fast Wave Concept Iterative Process) was made to extend the study of planar structures with thick flat conductor. This method is based on the concept of wave [9]. It is developed for the simple planar layer modeling of structures [10] or multi-layer [11], and even arbitrarily complex shape. This is an easy method to implement due to the absence of test functions. It is always convergent and has considerable execution speed due to the FMT (Fast Modal Transform).

\section{Iterative Method FWCIP}

This method is well suited to the calculation of planar structures. In fact, TE and TM modes are used in the iterative method as digital basis of spectral domain in which the FFT. Subsequently, the concept of fast wave is introduced to reflect the boundary conditions and continuity of relationships in different parts of the interface $\Omega$ in terms of waves.

The method involves determining an effective relationship to link the incident and reflected waves in different dielectric layers expressing thoughts in modal domain and the boundary conditions and continuity, expressed in terms of waves in spatial domain. The iterative process is then used to move from one field to another using the FMT thus accelerating the iterative process and then the convergence of the method. The use of the FMT requires the pixel description of the different regions of the dielectric interfaces. Thus the electromagnetic behavior of a planar structure will be described by writing the boundary conditions and continuity of the tangential fields on each pixel containing the interface circuitry to study. This integral formulation retains the advantages well known iterative methods including ease of implementation and speed of execution.

The flowchart in Figure 1 summarizes the evolution of the iterative method for a planar structure with three layers of different mediums [12].

The theoretical formulation for the iterative method is based on determining the relationship between the incident waves $\vec{A}_{1}, \vec{A}_{21}, \vec{A}_{22}$ and $\vec{A}_{3}$ defined in spatial domain and the reflected waves $\vec{B}_{1}, \vec{B}_{21}, \vec{B}_{22}$ and $\vec{B}_{3}$, defined in the spectral domain. The passage of the spatial domain to the spectral domain is using modal Fourier transform (FMT). The passage of the spectral domain to the space domain is using the transform inverse Fourier modal (FMT - 1). These operations are done with repetitions until the convergence of the method. FMT and FMT - 1 are used to speed up the computation time of the method.

$\hat{\Gamma}_{\Omega 1}$ and $\hat{\Gamma}_{\Omega 2}$ : Diffraction operators, giving the incident waves from the reflected waves that diffract at the discontinuities plans $\left(\Omega_{1}\right.$ and $\left.\Omega_{2}\right)$. They are defined in spatial domain and found in these image operators circuits placed at plans $\left(\Omega_{1}\right.$ and $\left.\Omega_{2}\right)$.

$\hat{\Gamma}_{k}$ : Operator reflection ensuring the link between the incident waves and the reflected waves. It is defined in the spectral domain. It contains information on the hous- 


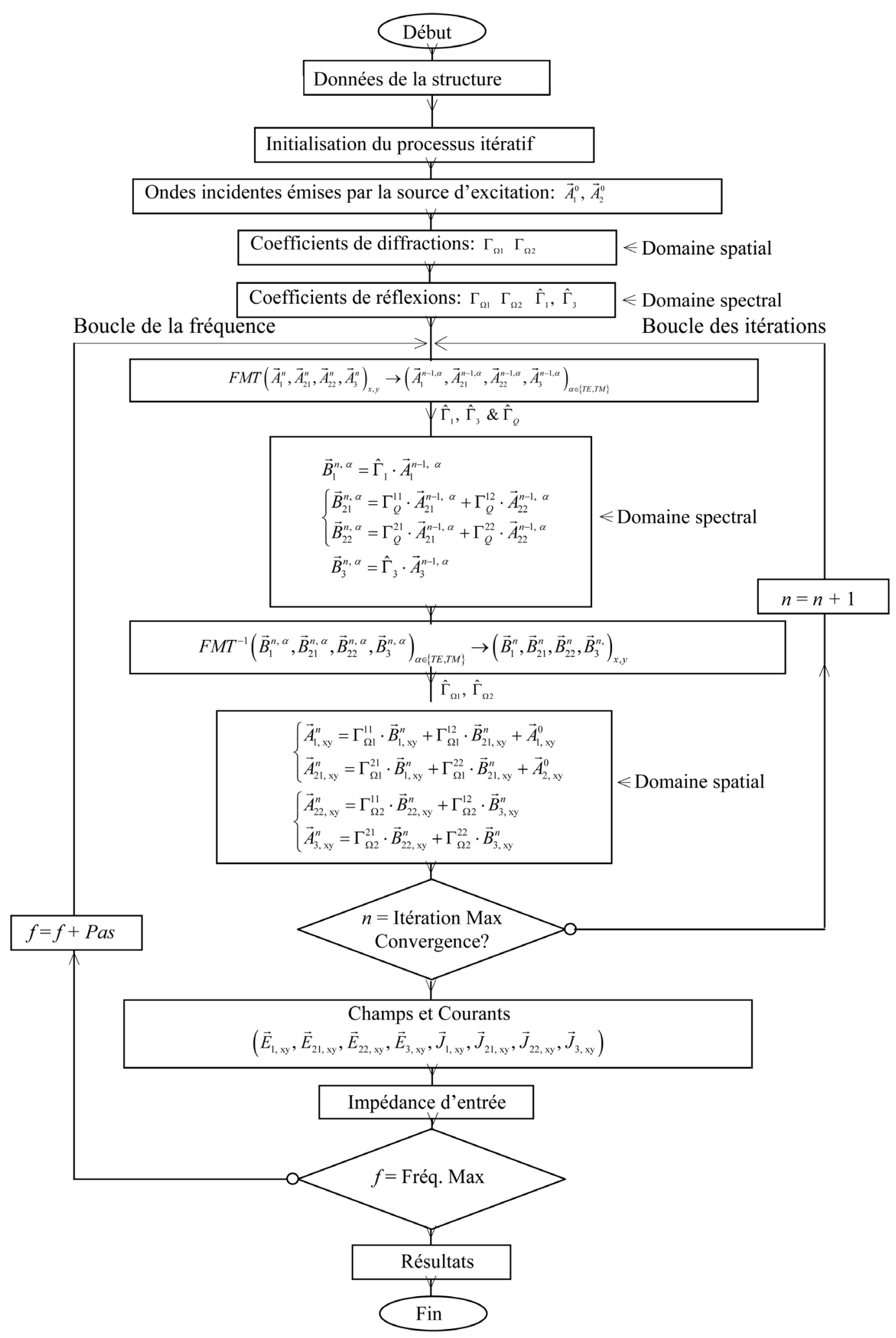

Figure 1. Flowchart summarizes the evolution of the iterative method for a planar structure with three layers of different mediums. It's available for every frequency and step in our case we took a max frequency equal to $20 \mathrm{GHz}$ and a step equal to 0.01 . 
ing walls and the relative permittivity of the different mediums of the structure, $k \in$ $\{$ medium 1, medium 3 \}.

$\hat{\Gamma}_{Q}$ : Diffraction Operator at each interface $\left(\Omega_{1}\right.$ and $\left.\Omega_{2}\right)$.

The evolution of iterations through the spectral domain to the space domain is done using the Fourier transform modal "FMT" which considerably reduces the calculation time. Modal Fourier transform requires the fragmentation of discontinuity planes $\left(\Omega_{1}\right.$ and $\Omega_{2}$ ) in pixels and this so that the electromagnetic behavior of the overall circuit will be summarized by writing the boundary conditions and continuity of the tangential fields on each pixel. The iterative process stops when it reaches the convergence of results.

The terms below link the incident waves " $\vec{A}_{1}, \vec{A}_{21}, \vec{A}_{22}$ and $\vec{A}_{3}$ " to the reflected waves " $\vec{B}_{1}, \vec{B}_{21}, \vec{B}_{22}$ and $\vec{B}_{3}$ " when they pass the space domain to the spectral domain:

$$
\begin{aligned}
& \left(\begin{array}{l}
A_{1} \\
A_{21}
\end{array}\right)=\hat{\Gamma}_{\Omega 1}\left(\begin{array}{l}
B_{1} \\
B_{21}
\end{array}\right)+\left(\begin{array}{l}
A_{1}^{0} \\
A_{2}^{0}
\end{array}\right) \\
& \left(\begin{array}{l}
B_{21} \\
B_{22}
\end{array}\right)=\hat{\Gamma}_{Q}\left(\begin{array}{l}
A_{21} \\
A_{22}
\end{array}\right) \\
& \left(\begin{array}{l}
A_{22} \\
A_{3}
\end{array}\right)=\hat{\Gamma}_{\Omega 2}\left(\begin{array}{c}
B_{22} \\
B_{3}
\end{array}\right) \\
& \left(\begin{array}{l}
\vec{J}_{21} \\
\vec{J}_{22}
\end{array}\right)=\left(\begin{array}{ll}
\hat{Y}_{11} & \hat{Y}_{12} \\
\hat{Y}_{21} & \hat{Y}_{22}
\end{array}\right)\left(\begin{array}{l}
\vec{E}_{21} \\
\vec{E}_{22}
\end{array}\right)
\end{aligned}
$$

The operators of diffraction $\hat{\Gamma}_{\Omega 1}$ and $\hat{\Gamma}_{\Omega 2}$ contains the images of circuit that being in the $\Omega_{1}$ and $\Omega_{2}$ plans.

- Diffraction Operator: $\hat{\Gamma}_{\Omega 1}$

For a source of bilateral excitation polarized in (oy), the overall diffraction operator is written from the diffraction operators in different regions of $\Omega_{1}$ plane (metal region, source region of excitement, dielectric region):

$$
\begin{aligned}
& {\left[\begin{array}{l}
\hat{\Gamma} \Omega\left(\begin{array}{l}
\mathrm{x} \\
\mathrm{y}
\end{array}\right)
\end{array}\right]}
\end{aligned}
$$

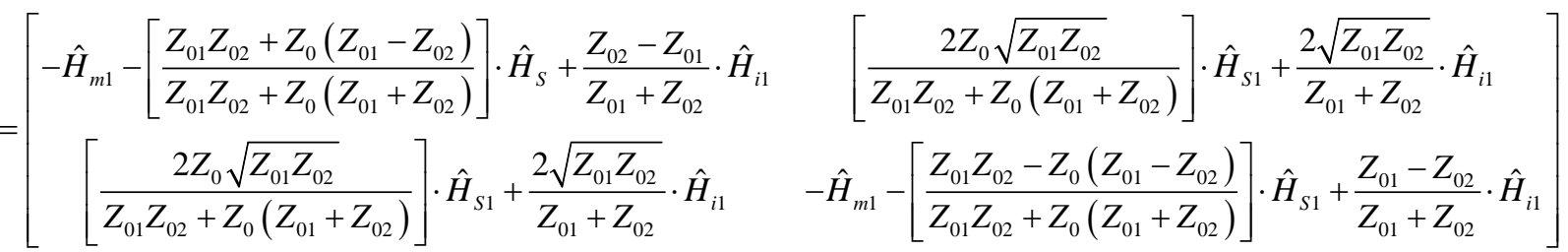

where: $H_{\mathrm{s} 1}=1$ on the source and 0 elsewhere.

$H_{\mathrm{m} 1}=1$ on the metal and 0 elsewhere.

$H_{\mathrm{i} 1}=1$ on the dielectric and 0 elsewhere.

- Diffraction Operator: $\hat{\Gamma}_{\Omega 2}$

The overall diffraction operator is written from the diffraction operators in different regions of $\Omega_{2}$ plane (metal region, dielectric region): 


$$
\left[\hat{\Gamma}_{\Omega 2\left(\begin{array}{c}
\mathrm{x} \\
\mathrm{y}
\end{array}\right)}\right]=\left[\begin{array}{cc}
-\hat{H}_{m 2}+\frac{Z_{02}-Z_{01}}{Z_{03}+Z_{02}} \cdot \hat{H}_{i 2} & \frac{2 \sqrt{Z_{03} Z_{02}}}{Z_{03}+Z_{02}} \cdot \hat{H}_{i 2} \\
\frac{2 \sqrt{Z_{03} Z_{02}}}{Z_{03}+Z_{02}} \cdot \hat{H}_{i 2} & -\hat{H}_{m 2}+\frac{Z_{03}-Z_{02}}{Z_{03}+Z_{02}} \cdot \hat{H}_{i 2}
\end{array}\right]
$$

where: $H_{m 2}=1$ on the metal and 0 elsewhere.

$H_{i 2}=1$ on the dielectric and 0 elsewhere

- Expression of the reflection operator: $\hat{\Gamma}_{k}$

It is defined in the spectral domain and contains information about the nature of the housing and the relative permittivity of the medium 1 and 3 of the structure. It is expressed by the following relationship:

$$
\hat{\Gamma}_{k}=\sum_{m, n, \alpha}\left|f_{m n}^{\alpha}\right\rangle \frac{1-Z_{0 k} Y_{m n}^{\alpha, k}}{1+Z_{0 k} Y_{m n}^{\alpha, k}}\left\langle f_{m n}^{\alpha}\right|
$$

$f_{m n}^{\alpha}$ : Basic functions. It depends on the nature of the box.

$Z_{0 k}=\frac{\eta}{\sqrt{\varepsilon_{r_{k}}}}:$ Impedance of the middle $k \in\{$ midium 1 , medium 2$\}$.

$\eta=120 \Pi$ : Vacuum impedance.

$Y_{m n}^{\alpha, k}$ : Mode admittance reduced to the level of $\Omega$ plan.

-For a top cover (or lower) placed at a distance $h$ from $\Omega$ plan.

$$
Y_{m n}^{\alpha, k}=Y_{m n}^{\alpha}\left(\varepsilon_{r_{k}}\right) \cdot \operatorname{coth}\left(\gamma_{m n}\left(\varepsilon_{r_{k}}\right) \cdot h\right)
$$

-For An open circuit without top cover (or lower).

$$
Y_{m n}^{\alpha, k}=Y_{m n}^{\alpha}\left(\varepsilon_{r_{k}}\right)
$$

$Y_{m n}^{\alpha}\left(\varepsilon_{r_{k}}\right):$ Mode admittance expressed by:

$$
\begin{aligned}
& T E: Y_{m n}^{T E}\left(\varepsilon_{r_{k}}\right)=\frac{\gamma_{m n}\left(\varepsilon_{r_{k}}\right)}{j \omega \mu_{0}} \\
& T M: Y_{m n}^{T M}\left(\varepsilon_{r_{k}}\right)=\frac{j \omega \varepsilon_{0} \varepsilon_{r_{k}}}{\gamma_{m n}\left(\varepsilon_{r_{k}}\right)}
\end{aligned}
$$

$\gamma_{m n}\left(\varepsilon_{r_{k}}\right):$ Propagation constant

$$
\gamma_{m n}^{2}\left(\varepsilon_{r_{k}}\right)=\left(\frac{m \Pi}{a}\right)^{2}+\left(\frac{n \Pi}{b}\right)^{2}-k_{0}^{2} \varepsilon_{r_{k}}
$$

$k_{0}=\frac{\omega}{c}:$ Wave number in a vacuum.

$c=\frac{{ }^{c} 1}{\sqrt{\varepsilon_{0} \mu_{0}}}:$ Speed of light $\left(3 \times 10^{8} \mathrm{~m} / \mathrm{s}\right)$

$m, n$ : Designating the index for modes $\in\{N\}$.

$\alpha$ : mode indicator $T E$ (Transverse Electric), $T M$ (Transverse Magnetic).

$k$. Medium considered $k \in\{1,2\}$.

$\varepsilon_{r_{k}}$ : Relative permittivity of the medium $k \in\{1,2\}$. 
$\varepsilon_{0}:$ Vacuum permittivity $(\mathrm{F} / \mathrm{m})$.

$\mu_{0:}$ Magnetic vacuum permeability $(\mathrm{H} / \mathrm{m})$.

$\omega$ : Angular pulsation equal to pulsation $2 \prod f(\mathrm{rd} / \mathrm{s})$.

\section{- Expression of the FMT}

The Fourier transform in cosine and sine is defined by:

$$
2 D-F F T_{\cos \sin }\left(\begin{array}{l}
E_{x}(i, j) \\
E_{y}(i, j)
\end{array}\right)=\left[\begin{array}{l}
\sum_{i=1}^{N_{01}} \sum_{j=1}^{N_{02}} E_{x}(i, j) \cdot \cos \left(\frac{m \Pi i}{N_{01}}\right) \cdot \sin \left(\frac{n \Pi j}{N_{02}}\right) \\
\sum_{i=1}^{N_{01}} \sum_{j=1}^{N_{02}} E_{y}(i, j) \cdot \sin \left(\frac{m \Pi i}{N_{01}}\right) \cdot \cos \left(\frac{n \Pi j}{N_{02}}\right)
\end{array}\right]
$$

The Fourier mode transform (FMT) is defined by:

$$
\left(\begin{array}{c}
e_{m n}^{T E} \\
e_{m n}^{T M}
\end{array}\right)=\hat{T} \cdot 2 D-F F T_{\cos \sin }\left(\begin{array}{l}
E_{x}(i, j) \\
E_{y}(i, j)
\end{array}\right)=\operatorname{FMT}\left(\begin{array}{l}
E_{x}(i, j) \\
E_{y}(i, j)
\end{array}\right)
$$

$\hat{T}$ : Passing modal operator in the area expressed by:

$$
\begin{gathered}
\hat{T}=K(m, n) \cdot\left[\begin{array}{cc}
\frac{n}{b} & -\frac{m}{a} \\
\frac{m}{a} & \frac{n}{b}
\end{array}\right] \\
K(m, n)=\frac{1}{\sqrt{\left(\frac{m}{a}\right)^{2}+\left(\frac{n}{b}\right)^{2}}} \cdot \sqrt{\frac{2 \sigma_{m n}}{a b}}
\end{gathered}
$$

\section{- The reflection operator of the Quadruple: $\hat{\Gamma}_{Q}$}

The reflection operator of the Quadruple is defined in layer 2 of the structure to be studied. It links the incident waves " $\vec{A}_{21}, \vec{A}_{22}$ ” the reflected waves " $\vec{B}_{21}, \vec{B}_{22}$ " they pass the space domain to the spectral domain. The quadruple $Q$ ensures the passage of plan $\Omega_{1}$ to plan $\Omega_{2}$ and inversely.

According to the diagram in Figure 1 we can write:

$$
\left(\begin{array}{l}
B_{21} \\
B_{22}
\end{array}\right)=\hat{\Gamma}_{Q}\left(\begin{array}{l}
A_{21} \\
A_{22}
\end{array}\right)
$$

- Parameters $\hat{Y}_{i j}$ du quadruple Q:

$$
\begin{array}{r}
\left\{\begin{array}{l}
J_{21}=Y_{11} E_{21}+Y_{12} E_{22} \\
J_{22}=Y_{21} E_{21}+Y_{22} E_{22}
\end{array}\right. \\
\left\{\begin{array}{l}
\overrightarrow{J_{0}}=\overrightarrow{J_{1}}+\overrightarrow{J_{21}} \\
\overrightarrow{J_{2}}=\overrightarrow{J_{22}}+\overrightarrow{J_{3}} \\
\overrightarrow{J_{1}}=Y_{h} \overrightarrow{E_{3}} \\
\overrightarrow{E_{1}}=\overrightarrow{E_{21}} \\
\overrightarrow{E_{22}}=\overrightarrow{E_{3}}
\end{array}\right.
\end{array}
$$

The symmetry of the structure, allows us to write: 


$$
\left\{\begin{array}{l}
\hat{Y}_{11}=\hat{Y}_{22} \\
\hat{Y}_{12}=\hat{Y}_{21}
\end{array}\right.
$$

After some mathematically manipulation, it is possible to determine the matrix:

$$
\hat{\Gamma}_{Q}=\frac{1}{C}\left[\begin{array}{cc}
1-\left(Y_{11} Z_{02}\right)^{2}+\left(Y_{12} Z_{02}\right)^{2} & -2 Y_{12} Z_{02} \\
-2 Y_{12} Z_{02} & 1-\left(Y_{11} Z_{02}\right)^{2}+\left(Y_{12} Z_{02}\right)^{2}
\end{array}\right]
$$

With: $\quad C=\left(1+Y_{11} Z_{02}\right)^{2}-\left(Y_{12} Z_{02}\right)^{2}$

$$
\begin{aligned}
& \Gamma_{Q}=\sum_{m, n, \alpha}\left[\begin{array}{ll}
\left|f_{m n}^{\alpha}\right\rangle \frac{1-\left(Y_{11} Z_{02}\right)^{2}+\left(Y_{12} Z_{02}\right)^{2}}{\left(1+Y_{11} Z_{02}\right)^{2}-\left(Y_{12} Z_{02}\right)^{2}}\left\langle f_{m n}^{\alpha}\right| & \left|f_{m n}^{\alpha}\right\rangle \frac{-2 Y_{12} Z_{02}}{\left(1+Y_{11} Z_{02}\right)^{2}-\left(Y_{12} Z_{02}\right)^{2}}\left\langle f_{m n}^{\alpha}\right| \\
\left|f_{m n}^{\alpha}\right\rangle \frac{-2 Y_{12} Z_{02}}{\left(1+Y_{11} Z_{02}\right)^{2}-\left(Y_{12} Z_{02}\right)^{2}}\left\langle f_{m n}^{\alpha}\right| & \left|f_{m n}^{\alpha}\right\rangle \frac{1-\left(Y_{11} Z_{02}\right)^{2}+\left(Y_{12} Z_{02}\right)^{2}}{\left(1+Y_{11} Z_{02}\right)^{2}-\left(Y_{12} Z_{02}\right)^{2}}\left\langle f_{m n}^{\alpha}\right|
\end{array}\right] \\
& \left\{\begin{array}{l}
B_{21}^{\alpha}=\sum_{m, n, \alpha}\left|f_{m n}^{\alpha}\right\rangle\left(\frac{1-\left(Y_{11} Z_{02}\right)^{2}+\left(Y_{12} Z_{02}\right)^{2}}{\left(1+Y_{11} Z_{02}\right)^{2}-\left(Y_{12} Z_{02}\right)^{2}}\right)^{\alpha}\left\langle f_{m n}^{\alpha}\left|A_{21}^{\alpha}+\sum_{m, n, \alpha}\right| f_{m n}^{\alpha}\right\rangle\left(\frac{-2 Y_{12} Z_{02}}{\left(1+Y_{11} Z_{02}\right)^{2}-\left(Y_{12} Z_{02}\right)^{2}}\right)^{\alpha}\left\langle f_{m n}^{\alpha}\right| A_{22}^{\alpha} \\
B_{22}^{\alpha}=\sum_{m, n, \alpha}\left|f_{m n}^{\alpha}\right\rangle\left(\frac{-2 Y_{12} Z_{02}}{\left(1+Y_{11} Z_{02}\right)^{2}-\left(Y_{12} Z_{02}\right)^{2}}\right)^{\alpha}\left\langle f_{m n}^{\alpha}\left|A_{21}^{\alpha}+\sum_{m, n, \alpha}\right| f_{m n}^{\alpha}\right\rangle\left(\frac{1-\left(Y_{11} Z_{02}\right)^{2}+\left(Y_{12} Z_{02}\right)^{2}}{\left(1+Y_{11} Z_{02}\right)^{2}-\left(Y_{12} Z_{02}\right)^{2}}\right)^{\alpha}\left\langle f_{m n}^{\alpha}\right| A_{21}^{\alpha}
\end{array}\right.
\end{aligned}
$$

$f_{m n}^{\alpha}$ : Bases function of the box modes.

With $\alpha \in\{T E, T M\}$.

\section{Formulation of the Problem}

To show the robustness of this new formulation of the iterative method we have applied to the study of two coupled micro strip lines, parallel, symmetrical and placed in the same plane. Metal foils which constitute them are copper and have a thickness which is important. The structure of these is presented below in Figure 1 and Figure 2. This structure is the basic element of a band-pass filter or band cut made in coplanar technology. This filtering function can be encountered in all the wireless emission-receiving systems.

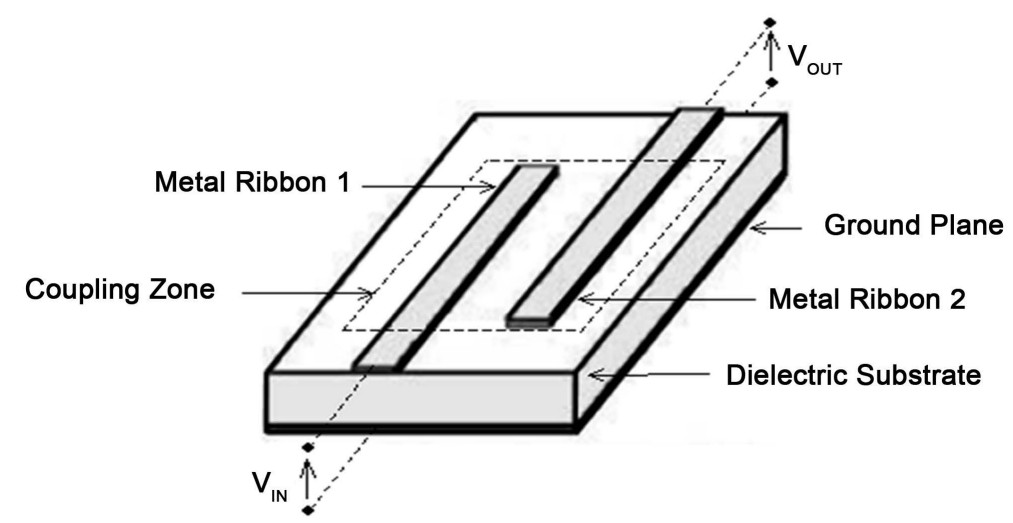

Figure 2. Two coplanar and symmetrical lines. 
In our model both input and output voltage "VIN et VOUT" of the structure to study will be modeled by two field sources $S_{1}\left\{E_{1}, J_{1}\right\}$ and $S_{2}\left\{E_{2}, J_{2}\right\}$.

\subsection{Electromagnetic Model of the Study Structure}

Figure 3(a) shows the structure to study and Figure 3(b) shows the electromagnetic model we propose to study this structure. The latter consists in three layers of different materials. The first layer is filled with a dielectric substrate, assumed without loss, relative permittivity $\varepsilon_{\mathrm{r} 1}$ and thickness h1. It is placed between the ground plane and the plane $\Omega_{1}$ discontinuity. The second layer thickness h2 of which is equal to the thickness $\mathrm{T}$ of the formants metal strips the two coplanar lines. It is placed between the two plans of $\Omega_{1}$ and $\Omega_{2}$ discontinuities and consisting of a complex effective permittivity $\tilde{\varepsilon}_{\text {eff }}$ medium modeling the different materials (copper and Air) formants this medium. The third layer is filled with air. It is placed between the $\Omega_{2}$ discontinuity plane and the top cover of the metal housing which surrounds the entire structure. The use of a metal case is necessary for reasons of shielding and modeling.

Structural parameters: $a=4.754 \mathrm{~mm}, \quad b=4.754 \mathrm{~mm}, c_{1} \approx 0.26 \mathrm{~mm}$, $d_{1} \approx 0.037 \mathrm{~mm}, \quad s \approx 0.074 \mathrm{~mm}, \quad L \approx 3.53 \mathrm{~mm}, \quad h_{1}=0.254 \mathrm{~mm}, h_{2}=T=10 \mu \mathrm{m}$, $h_{3}=1.99 \mathrm{~mm}, \varepsilon_{r 1}=9.9, \varepsilon_{r 2}=\tilde{\varepsilon}_{e f f}, \varepsilon_{r 3}=1, \sigma=59.6 \times 10^{6} \mathrm{~S} / \mathrm{m}$.

The plan of discontinuity $\Omega_{1}$ contains the input and the output of the circuit and the two metal ribbons, without thickness, modeling the undersides of the two micro strip lines. The $\Omega_{2}$ plan only contains two metal ribbons, without thickness, modeling the upper faces of the two micro strip lines (Figure 4 and Figure 5).

\subsection{Technical Calculation $Y_{i j}$ Parameters of the Coupling Matrix between the Sources of Excitations $S_{1}\left(E_{1}, J_{1}\right)$ and $S_{2}\left(E_{2}, J_{2}\right)$.}

We present in Figure 6 the technique for calculating admittances $Y_{i j}$ parameters of the coupling matrix between the different sources of excitations of the study structure. This technique allows the electromagnetic calculation of equivalent quadruple source view of

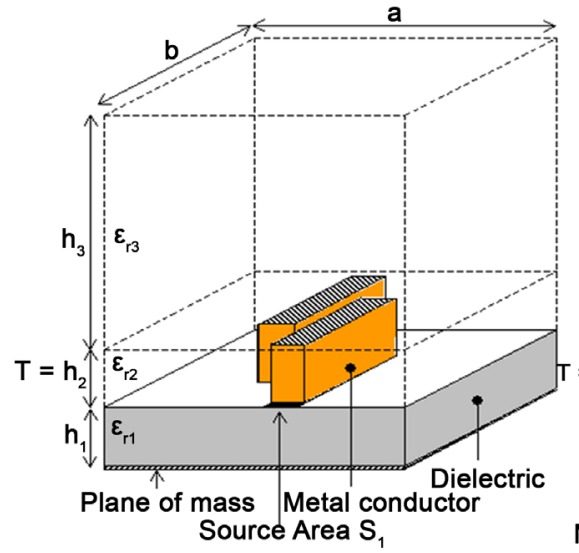

(a)

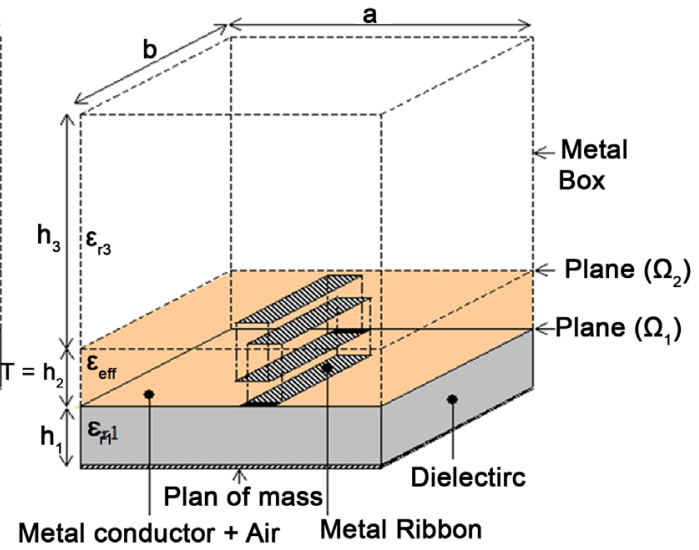

(b)

Figure 3. (a): Studied structure; (b): Electromagnetic model. 


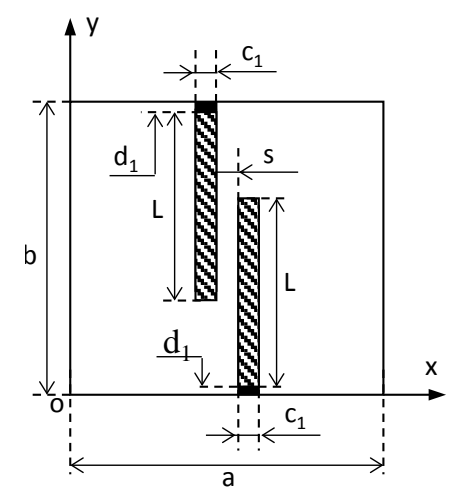

(a)

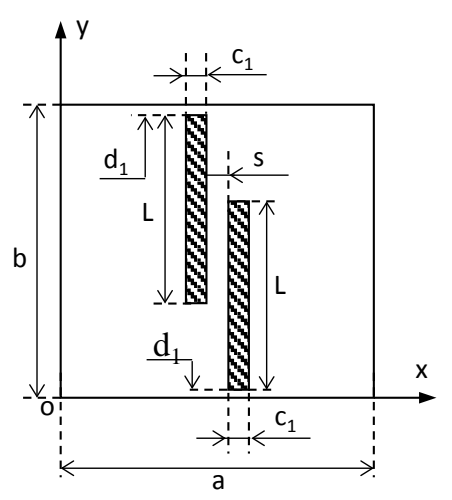

(b)

Figure 4. Quotes of discontinuities plans. (a): Plan $\Omega_{1}$; (b): Plan $\Omega_{2}$.

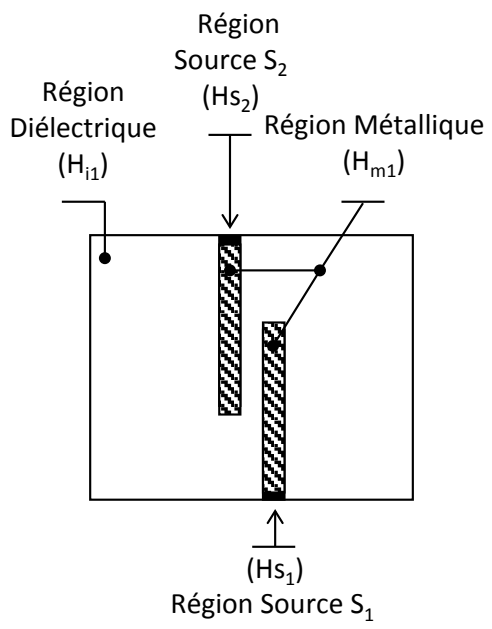

(a)

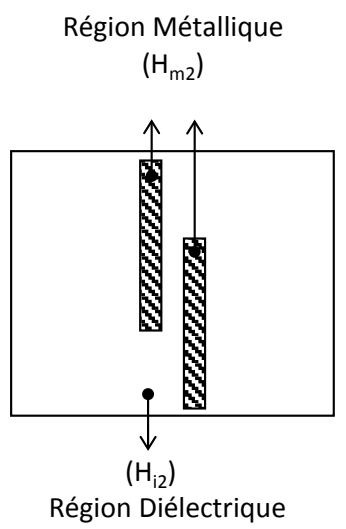

(b)

Figure 5. Definition of the different regions of the discontinuities plans (a): Plan $\Omega_{1}$; (b): Plan $\Omega_{2}$.

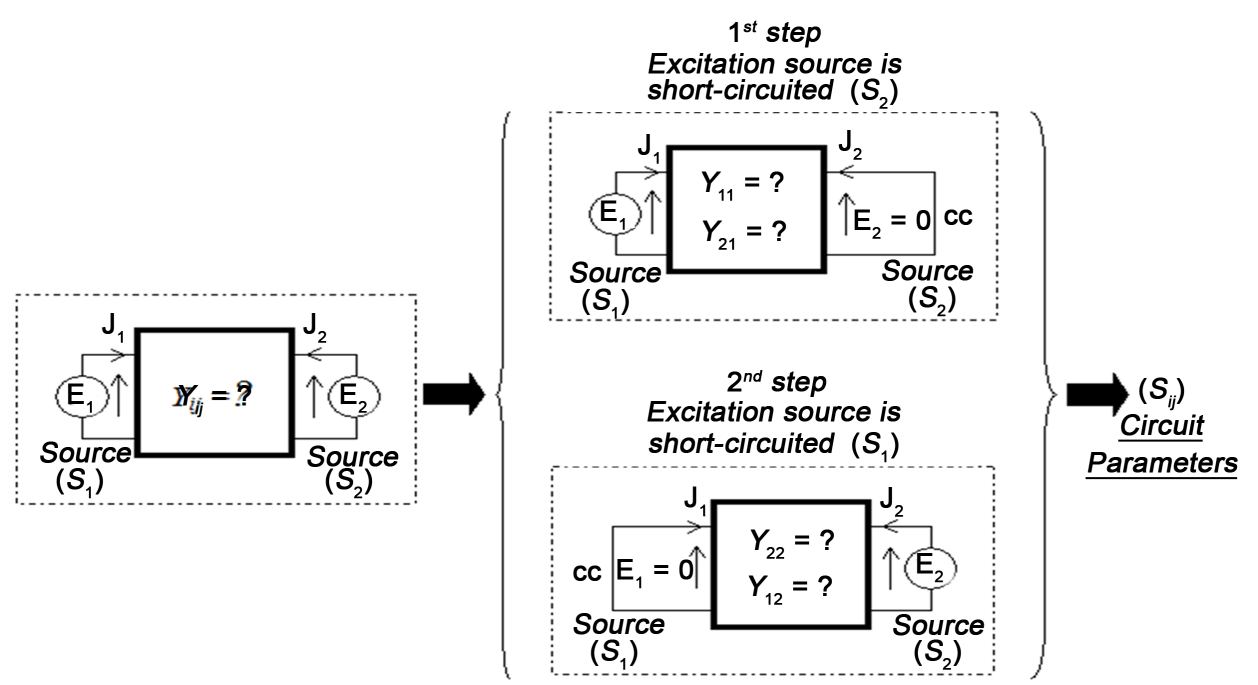

Figure 6. Technical way of calculating $Y_{i j}$ admittances parameters of the coupling matrix between $S_{1}\left(E_{1}, J_{1}\right)$ et $S_{2}\left(E_{2}, J_{2}\right)$. 
excitations considered $S_{1}\left(E_{1}, J_{1}\right)$ et $S_{2}\left(E_{2}, J_{2}\right)$. In this technique we apply the superposition theorem that considers the study structure is alternately excited by the source $S_{1}$ $\left(E_{1}, J_{1}\right)$ then by the source $S_{2}\left(E_{2}, J_{2}\right)$. This brings us to a problem with a single excitation source whose theoretical development is simple to prepare.

The study of structure (Figure $7(\mathrm{a})$ ) is energized by two sources localized $S_{1}\left(E_{1}, J_{1}\right)$ and $S_{2}\left(E_{2}, J_{2}\right)$. This sets the quadruple coupling shown in Figure 7(b).

- 1st Step: The localized source $S_{1}\left(E_{1}, J_{1}\right)$ is activated.

In this step we short circuit the excitation source $\mathrm{n}^{\circ} 2\left(E_{2}=0\right)$, as shown in Figure 8 we calculate input admittance seen by the excitation source $\mathrm{n}^{\circ} 1$ " $Y_{11}$ " and the transfer admittance (or coupling) of the $\mathrm{n}^{\circ} 1$ source to the source $\mathrm{n}^{\circ} 2$ " $Y_{21}$ ".

The matrix representation (1) allows us to write:

$$
\begin{gathered}
\left\{\begin{array}{l}
J_{1}=Y_{11} E_{1}+Y_{12} E_{2} \\
J_{2}=Y_{21} E_{1}+Y_{22} E_{2}
\end{array}\right. \\
E_{2}=0 \Rightarrow\left\{\begin{array}{l}
J_{1}=Y_{11} E_{1} \Rightarrow Y_{11}=\frac{J_{1}}{E_{1}} \\
J_{2}=Y_{21} E_{2} \Rightarrow Y_{21}=\frac{J_{2}}{E_{2}}
\end{array}\right.
\end{gathered}
$$

$J_{1}$ and $J_{2}$ current densities are created by the excitation source $\left\{S_{1}\right\}$ respectively at the source $\left\{S_{1}\right\}$ and location of the source shorted $\left\{S_{2}\right\}$. So that the parameters $Y_{11}$ and $Y_{21}$

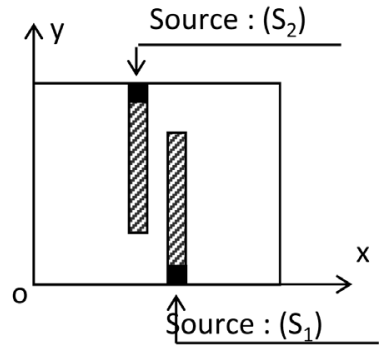

(a)

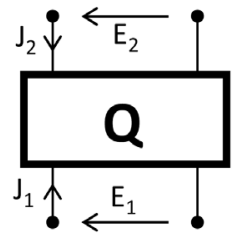

(b)
Coupling matrix enter $\left\{S_{1}\right.$ et $\left.S_{2}\right\}$

$\left(\begin{array}{l}J_{1} \\ J_{2}\end{array}\right)=\left(\begin{array}{ll}Y_{11} & Y_{12} \\ Y_{21} & Y_{22}\end{array}\right) \cdot\left(\begin{array}{l}E_{1} \\ E_{2}\end{array}\right)$

Figure 7. (a) Study Structure excited by two sources $\left\{S_{1}\right.$ et $\left.S_{2}\right\}$; (b) Coupling quadrupole between enter $\left\{S_{1}\right.$ et $\left.S_{2}\right\}$.

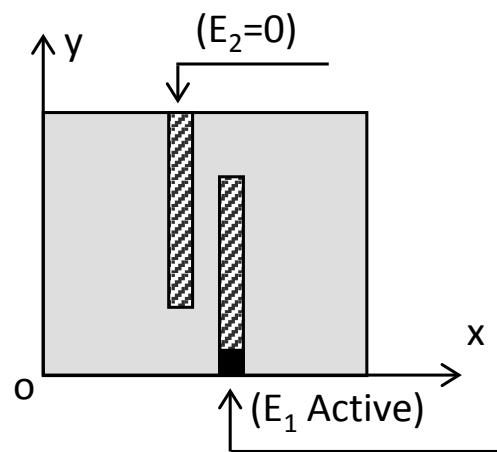

Figure 8. Study Structure excited by the source $\{\mathrm{S} 1\}$. 
are respectively the admittance seen by excitation source $\left\{S_{1}\right\}$ and the admittance viewpoints occupied by the source shorted $\left\{S_{2}\right\}$.

- 2nd Step: The localized source $S_{2}\left(E_{2}, J_{2}\right)$ is activated

In this second step we short-circuit the source $\mathrm{n}^{\circ} 1\left(E_{1}=0\right)$, as shown in Figure 9 we calculate the input admittance seen by the excitation source $\mathrm{n}^{\circ} 2$ " $Y_{22}$ " and the transfer admittance (or coupling) from source 2 to source $\mathrm{n}^{\circ} 1$ " $Y_{12}$ ".

The matrix representation (1) allows us to write:

$$
\begin{gathered}
\left\{\begin{array}{l}
J_{1}=Y_{11} E_{1}+Y_{12} E_{2} \\
J_{2}=Y_{21} E_{1}+Y_{22} E_{2}
\end{array}\right. \\
E_{1}=0 \Rightarrow\left\{\begin{array}{l}
J_{1}=Y_{12} E_{2} \Rightarrow Y_{12}=\frac{J_{1}}{E_{2}} \\
J_{2}=Y_{22} E_{2} \Rightarrow Y_{22}=\frac{J_{2}}{E_{2}}
\end{array}\right.
\end{gathered}
$$

$J_{1}$ and $J_{2}$ current densities are created by the excitation source $\left\{S_{2}\right\}$ respectively at source level $\left\{S_{2}\right\}$ and the location of the source shorted $\left\{S_{1}\right\}$. So that the parameters $Y_{22}$ and $Y_{12}$ are respectively the admittance seen by excitation source $\left\{S_{2}\right\}$ and the admittance viewpoints occupied by the source shorted $\left\{S_{1}\right\}$.

The calculation of parameters $Y_{i j}$ coupling matrix requires a convergence study of these items based on iterations, optimizing the computation time and increasing the accuracy of these results. By observing Figure 10 and Figure 11 we find that the number of iterations required for convergence of admittances $Y_{11}, Y_{12}, Y_{21}$ and $Y_{22}$ is 4000 iterations for a frequency $f=5 \mathrm{GHZ}$. This frequency is far from the resonance of the structure where we have maximum energy. At this level it takes a lot more iterations to reach convergence (about 20,000 iterations). Given the symmetry of the structure we find that: $Y_{11}=Y_{22}$ and $Y_{21}=Y_{12}$.

Figure 12 and Figure 13 respectively show changes depending on the frequency of the input admittance seen by the excitation source $\mathrm{n}^{\circ} 1$ " $Y_{11}$ " and the transfer admittance (or coupling) of the source $\mathrm{n}^{\circ} 1$ to the source $\mathrm{n}^{\circ} 2$ " $Y_{21}$ ". The validation of these results was carried out by comparison with those calculated by Ansoft HFSS software. This comparison shows that both results have the same variations and coincide with the 7.6 GHZ frequency. We notice a discrepancy between our results and those of HFSS software in the order of $4 \%$.

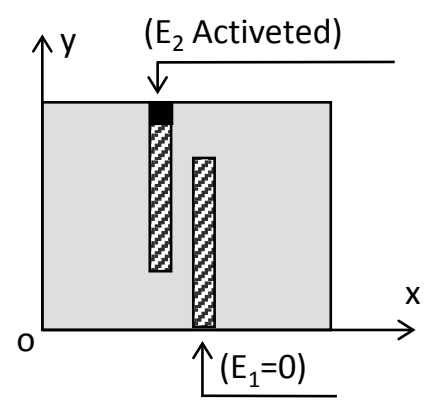

Figure 9. Studied structure excited by the source $\{$ S2 $\}$. 


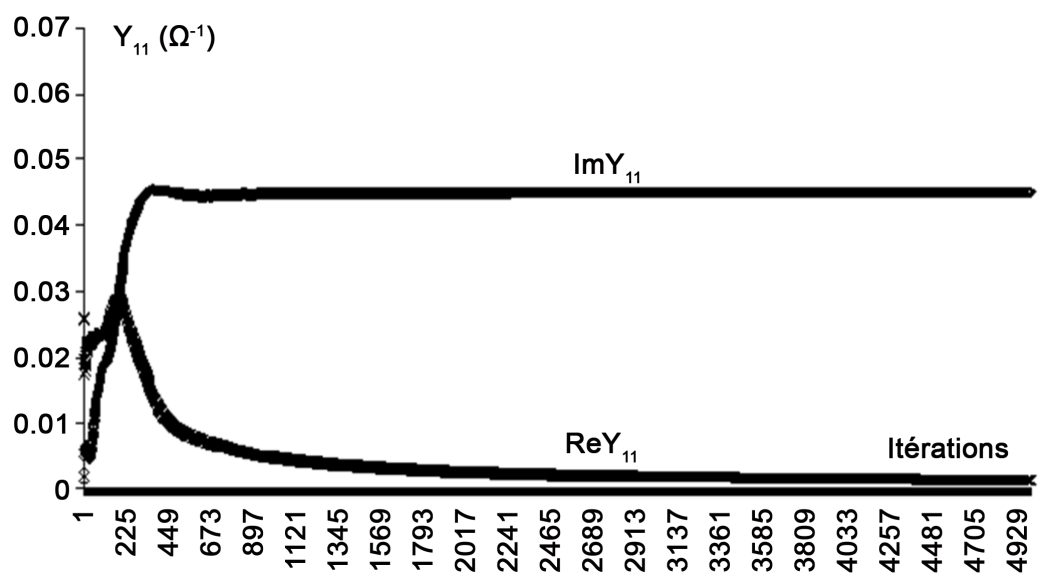

Figure 10. Input admittance $Y_{11}$ convergence based on iterations for ( $f=5 \mathrm{GHZ}$ ).

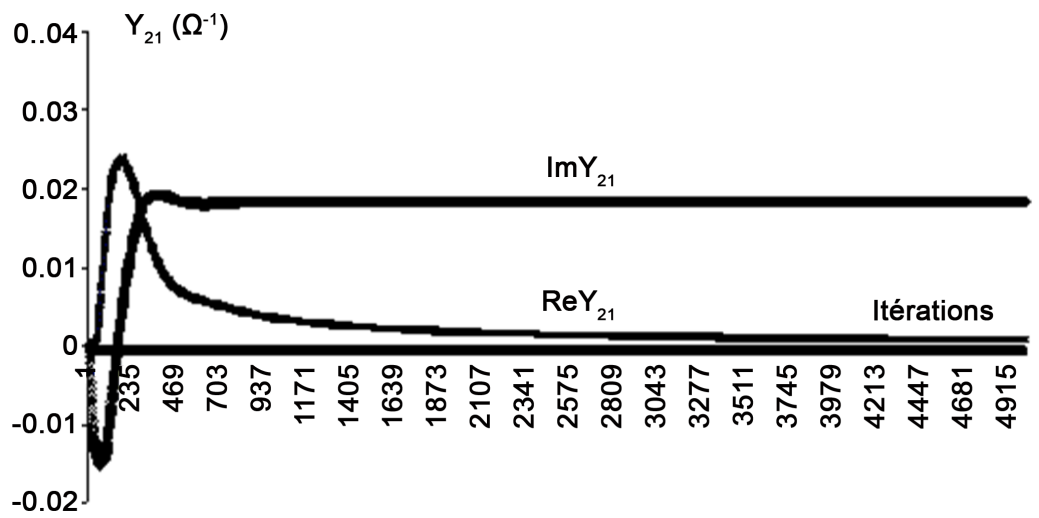

Figure 11. Coupling admittance convergence $Y_{21}$ based on iterations for $(f=5 \mathrm{GHZ})$.

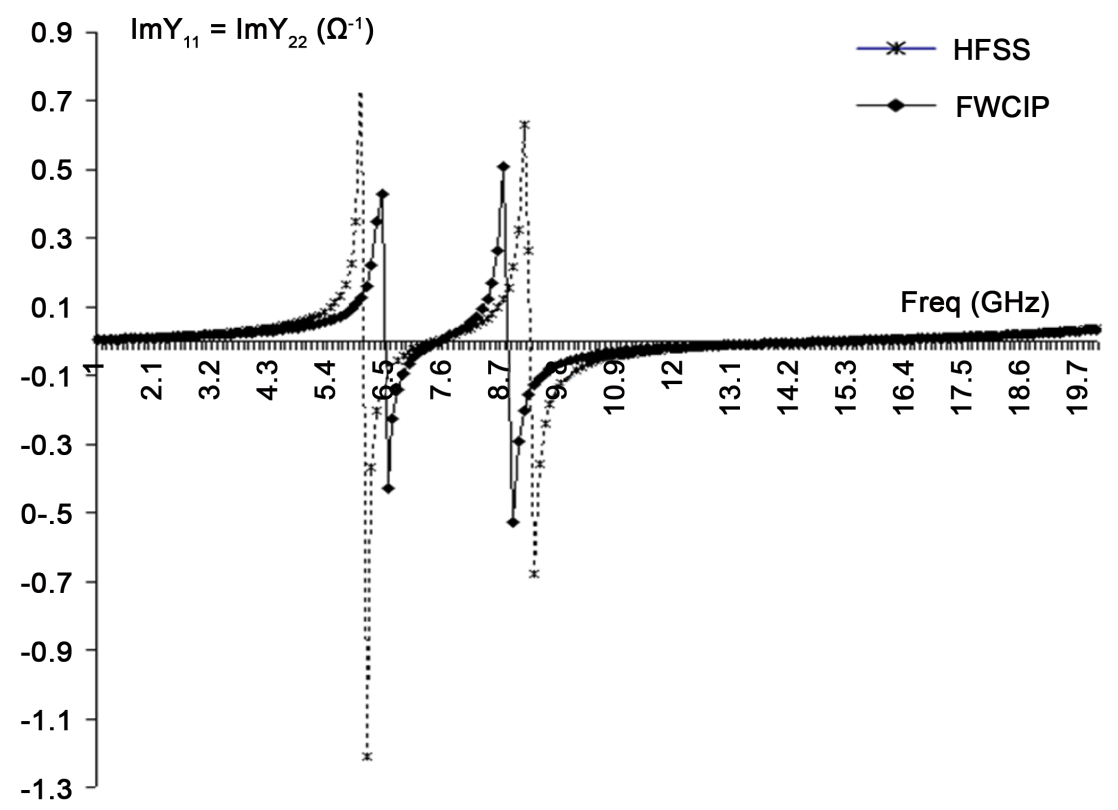

Figure 12. Input admittance $Y_{1 L}$ depending on the frequency (number of iterations: 5000). 


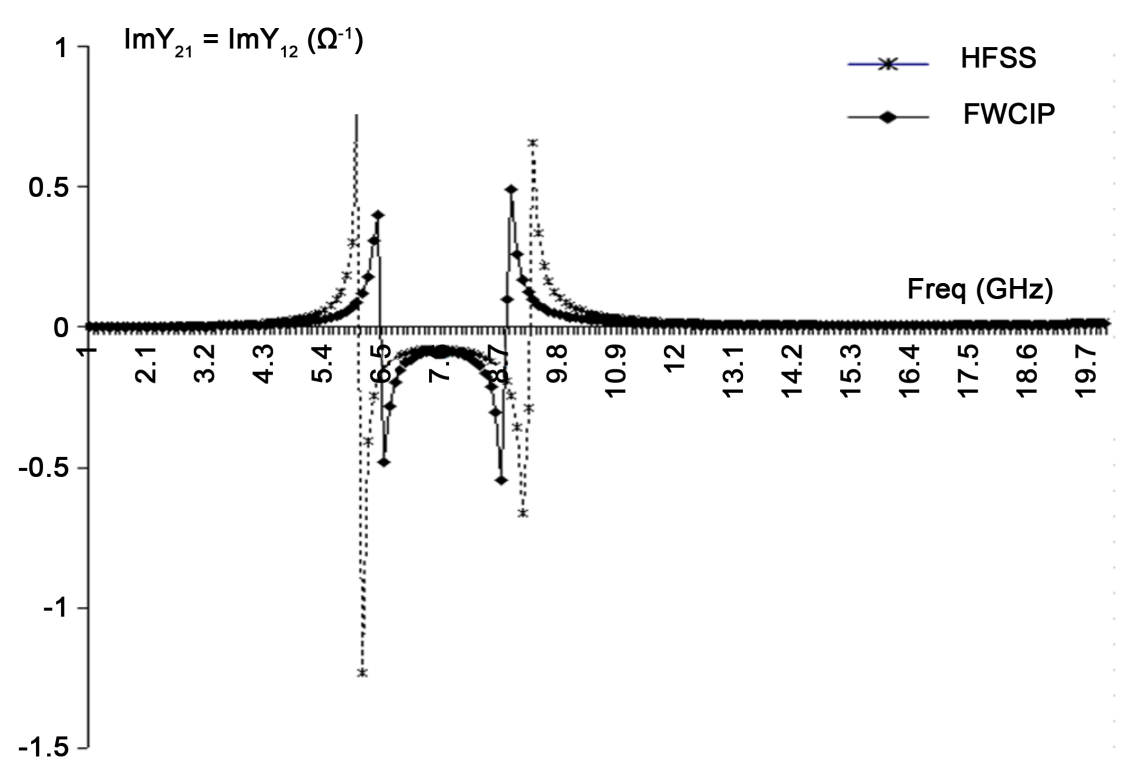

Figure 13. Transfer admittance (or coupling) $Y_{21}$ according to the frequency (number of iterations: 5000).

Admittances $Y_{11}$ and $Y_{22}$, are identical, this is translated by the symmetry of the structure, same for the admittances $Y_{12}$ and $Y_{21}$.

\subsection{Calculating $S_{i j}$ Parameters of the Study Structure}

The parameters $Y_{i j}$ of the coupling matrix between the two circuit excitation sources have been found. It remains to calculate the characteristic impedance of the study structure is necessary for the calculation of $S_{i j}$ parameters.

\subsubsection{Calculation of Characteristic Impedance ZC of the Study Structure}

We applied in a first step the empirical formulas of Hammerstad (based GARDIOL) to calculate the characteristic impedance of the study structure. These formulas we offer values approaching $Z_{c}$ For more details on the characteristic impedance must use a numerical method for the calculation.

$\varepsilon_{r}$ is the relative permittivity of the dielectric which is the speeding of the electromagnetic wave. By cons in a microstrip line (Figure 14(a)), the speeding is done in two different environments $\varepsilon_{r}$ "dielectric and the air". To simplify the problem we must determine an equivalent dielectric constant $\varepsilon_{\text {eff }}$ (Figure 14(b)), this model based on $\varepsilon_{r}$ and $h$.

$$
\begin{aligned}
& \lambda_{g}=\frac{\lambda_{0}}{\sqrt{\varepsilon_{e}}} \\
& \lambda_{0}=\frac{C}{f} \\
& Z_{c}=\frac{Z_{0}}{\sqrt{\varepsilon_{e}}}
\end{aligned}
$$




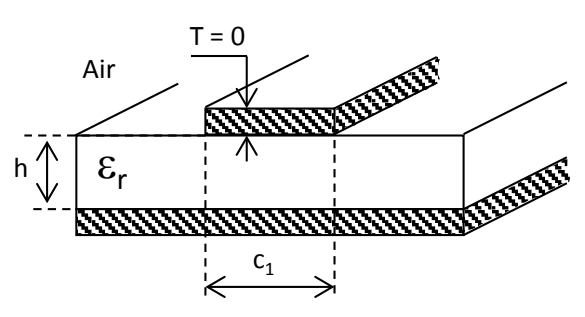

(a)

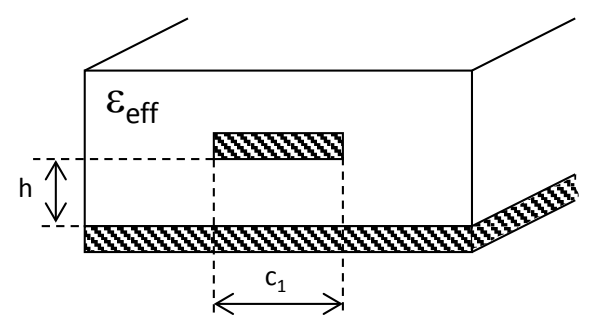

(b)

Figure 14. A: Real micro strip Line, B: fictive micro strip Line.

- For the microstrip line such as: $\frac{c_{1}}{h} \leq 1 \Rightarrow$

$$
\begin{gathered}
Z_{c}=\frac{60}{\sqrt{\varepsilon_{e}}} \log _{e}\left(\frac{8 \cdot h}{c_{1}}+\frac{c_{1}}{4 \cdot h}\right) \\
\varepsilon_{\text {eff }}=\frac{\varepsilon_{r}+1}{2}+\frac{\varepsilon_{r}-1}{2}\left[\left(1+\frac{12 \cdot h}{c_{1}}\right)^{-\frac{1}{2}}+0.04 \cdot\left(1-\frac{c_{1}}{h}\right)^{2}\right]
\end{gathered}
$$

- For microstrip line such as: $\frac{c_{1}}{h}>1 \Rightarrow$

$$
\begin{gathered}
Z_{c}=\frac{120 \cdot \Pi}{\sqrt{\varepsilon_{e}}}\left[\frac{c_{1}}{h}+1.393+0.667 \cdot \log _{e}\left(\frac{c_{1}}{h}+1.444\right)\right]^{-1} \\
\varepsilon_{\text {eff }}=\frac{\varepsilon_{r}+1}{2}+\frac{\varepsilon_{r}-1}{2}\left(1+\frac{12 \cdot h}{c_{1}}\right)^{-\frac{1}{2}}
\end{gathered}
$$

With: $\lambda_{g}$ guided wavelength.

$\lambda_{0}$ : wavelength in free space.

$\varepsilon_{\text {eff: }}$ effective permittivity of the micro strip line.

$C=2.99792458 \times 10^{8} \mathrm{~m} \cdot \mathrm{s}^{-1}$ : speed of light.

f. working frequency.

$Z_{c}$ : Characteristic impedance of the actual micro strip line.

$Z_{0}$ : Impedance characteristic of the imaginary line.

Adjusting for frequency:

It is possible to consider an approximate way, replacing $\varepsilon_{\text {eff }}$ in formulas by $\varepsilon_{e f f}(f)$.

$$
\varepsilon_{\text {eff }}(f)=\varepsilon_{r}-\frac{\varepsilon_{r}-\varepsilon_{\text {eff }}}{1+G \cdot\left(f / f_{d}\right)^{2}}
$$

With:

$$
f_{d}=\frac{1}{2 \cdot \mu_{0}} \frac{Z_{c}}{h} \text { et } G=0.6+0.009 \cdot Z_{c}
$$

Si $f \ll f_{d}$, it is not necessary to perform the correction.

For our application, the study structure has a ratio between the width of the metal band " $c_{1}$ " and the dielectric thickness " $h_{1}$ " equal to 1.023622 . 


$$
\frac{c_{1}}{h_{1}}=1.023622>1 \Rightarrow Z_{C}=48.25 \Omega
$$

The relative error obtained with respect to the characteristic impedance calculated using the ADS Linecalc tool (which is equal to $47,618 \Omega$ ) is equal to $1.3 \%$ This is for equal work often $13.5 \mathrm{GHZ}$.

\subsubsection{Calculation of $S_{i j}$ Parameters of the Study Structure}

The $Y_{i j}$ parameters of the structure study were calculated and so does the characteristic impedance $Z_{c}$. This allows to deduce the $S_{i j}$ parameters of the two micro strip lines coplanar relationship defined below:

$$
\begin{gathered}
S_{11}=\frac{\left(1-Z_{c} \cdot Y_{11}\right)\left(1+Z_{c} \cdot Y_{22}\right)+Z_{c}^{2} \cdot Y_{12} Y_{21}}{\left(1+Z_{c} \cdot Y_{11}\right)\left(1+Z_{c} \cdot Y_{22}\right)-Z_{c}^{2} \cdot Y_{12} Y_{21}} \\
S_{21}=\frac{-2 \cdot Z_{c} \cdot Y_{21}}{\left(1+Z_{c} \cdot Y_{11}\right)\left(1+Z_{c} \cdot Y_{22}\right)-Z_{c}^{2} \cdot Y_{12} Y_{21}} \\
S_{22}=\frac{\left(1+Z_{c} \cdot Y_{11}\right)\left(1-Z_{c} \cdot Y_{22}\right)+Z_{c}^{2} \cdot Y_{12} Y_{21}}{\left(1+Z_{c} \cdot Y_{11}\right)\left(1+Z_{c} \cdot Y_{22}\right)-Z_{c}^{2} \cdot Y_{12} Y_{21}} \\
S_{12}=\frac{-2 \cdot Z_{c} \cdot Y_{12}}{\left(1+Z_{c} \cdot Y_{11}\right)\left(1+Z_{c} \cdot Y_{22}\right)-Z_{c}^{2} \cdot Y_{12} Y_{21}}
\end{gathered}
$$

Calculating the parameters in decibel $S_{i j}$ is done by applying Equation (14) below:

$$
S_{i j}(\mathrm{~dB})=20 \cdot \log _{10} \sqrt{\operatorname{Re}\left(S_{i j}\right)^{2}+\operatorname{Im}\left(S_{i j}\right)^{2}}
$$

We present in the following, the effect of the error obtained in the approximate calculation of the characteristic impedance of the parameters $S_{i j}$

Formulas Hammerstad:

$$
Z_{c}=48.25 \Omega \Rightarrow S_{11}=-1.959552 \mathrm{~dB} \text { et } S_{21}=-4.861236 \mathrm{~dB}
$$

LineCalc of ADS:

$$
Z_{c}=48.618 \Omega \Rightarrow S_{11}=-1.919848 \mathrm{~dB} \text { et } S_{21}=-4.935580 \mathrm{~dB}
$$

Error:

Error on $Z_{c}=1.3 \%$, Error on $S_{11}=2 \%$, Error on $S_{21}=1.5 \%$.

An error of $1.3 \%$ on the characteristic impedance results in an error of $2 \%$ on the coefficient of reflection $S_{11}$ and an error of $1.5 \%$ on the transmission coefficient $S_{21}$. This for equal work often $13.5 \mathrm{GHZ}$ and 5000 iterations. We note that there is a slight increase of this error on the $S_{i j}$ parameters relative error on $Z_{c}$

Figure 15 and Figure 16 respectively show the variations in the transmission coefficients " $S_{21}$ " and reflection " $S_{11}$ " based on the frequency. Also view the symmetry of the structure we have obtained " $S_{11}=S_{22}$ " and " $S_{21}=S_{12}$ ". These results noted a strong coupling between the two sources of excitation in the frequency band " $41.6 \mathrm{GHZ}-42$ GHZ" where the coefficients of transmissions " $S_{21}$ and $S_{12}$ " are strictly greater than -3 $\mathrm{dB}-3 \mathrm{~dB}$ and reflection coefficients " $S_{11}$ et $S_{22}$ " are strictly less than $-10 \mathrm{~dB}$. In this frequency band the circuit is well adapted on both sides and it behaves as a band pass filter 


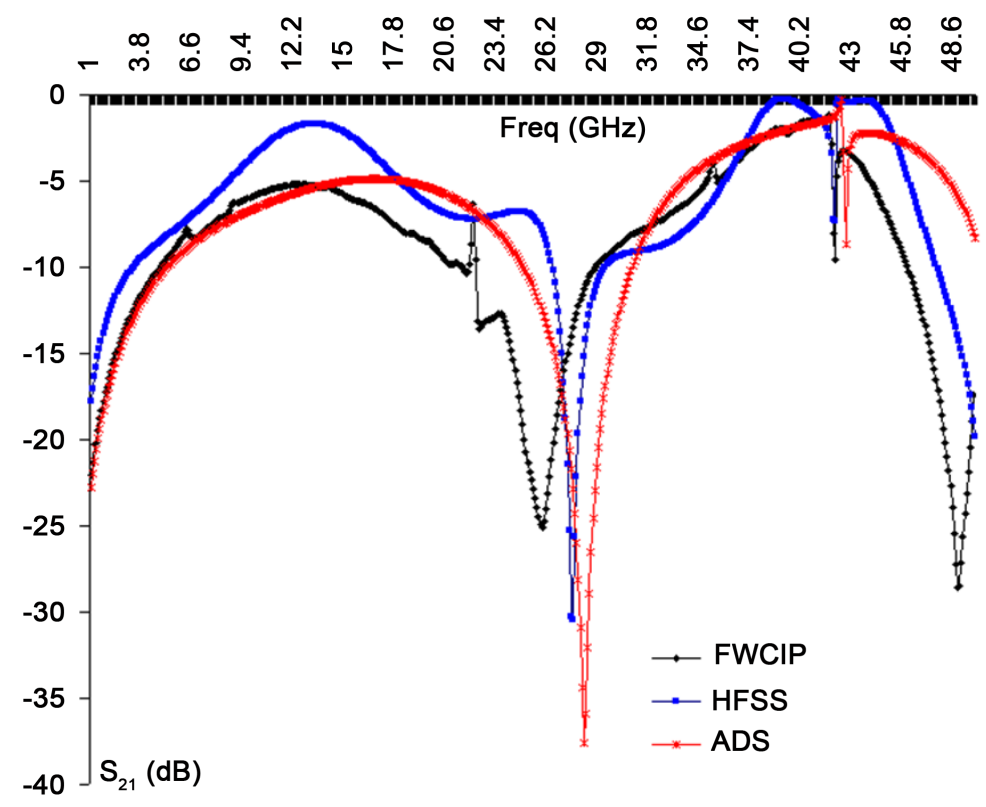

Figure 15. Transmission coefficient as a function of the frequency (Number of iterations: 1000).

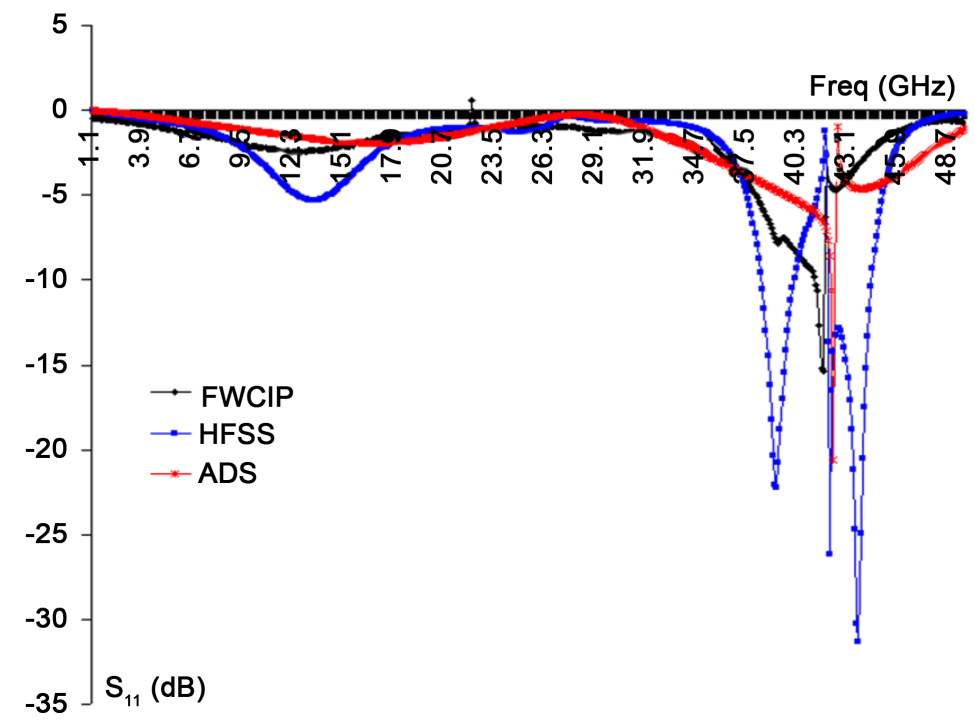

Figure 16. Reflection coefficient as a function of the frequency (Number of iterations: 1000).

By cons in the frequency band " $22.4 \mathrm{GHZ}-28.8 \mathrm{GHZ}$ " coupling is very small between the two sources of excitations. In this frequency band the coefficients of transmissions " $S_{21}$ and $S_{12}$ " are strictly less than $-10 \mathrm{~dB}$ " $S_{11}$ and $S_{22}$ " and coefficients of reflections are strictly greater than $-1.2 \mathrm{~dB}$. The circuit mismatched with the two inputs and it behaved like a notch filter.

The validation of these results was carried out by comparison with those calculated by Ansoft HFSS software and technology Agilent ADS software. This comparison shows that these results present the same variations and coincide in certain frequency bands. 
We report in Figure 17 the variation of the according transmission coefficient of the thickness $\mathrm{T}$ of the metal strip. This for equal work often $13.5 \mathrm{GHZ}$ and 5000 iterations. This curve shows that if we increase the thickness of the metal strips the transmission between the two sources of excitations increases, which is quite normal and valid even used the method of analysis. For a strictly smaller thickness $1 \mu \mathrm{m}$ we note that the variation is very low and the metal ribbons behave like metal strips without thickness. The beginning of the answer starts from $1 \mu \mathrm{m}$ and lasts up to $60 \mu \mathrm{m}$ where we notice a marked increase in transmittance with the thickness T. while increasing beyond $60 \mu \mathrm{m}$, we notice a saturation which is due probably to the cancellation of the edge effect. We will have a planar waveguide behavior that is different from two thin lines. The energy propagation is thus made according to this waveguide which is well known and characterized by its propagation constant " $\beta=\omega / c$ " which is linear and its characteristic impedance which is defined by:

$$
Z_{C}=\sqrt{\frac{\mu_{0}}{\varepsilon_{0}}}
$$

Figure 18 shows the variation of the transmission coefficient as a function of the thickness $\mathrm{T}$ of the metal strip and as a function of frequency. This is for 5000 iterations.

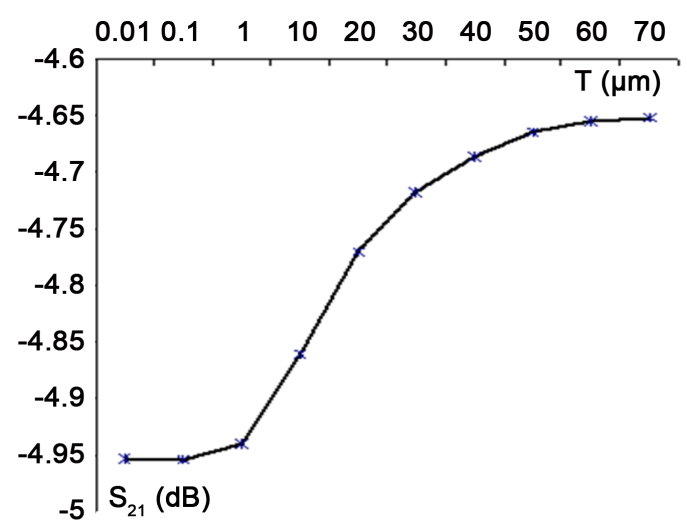

Figure 17. Variation of the transmission coefficient " $S_{21}$ " based on the thickness $T$ of the metal strip.

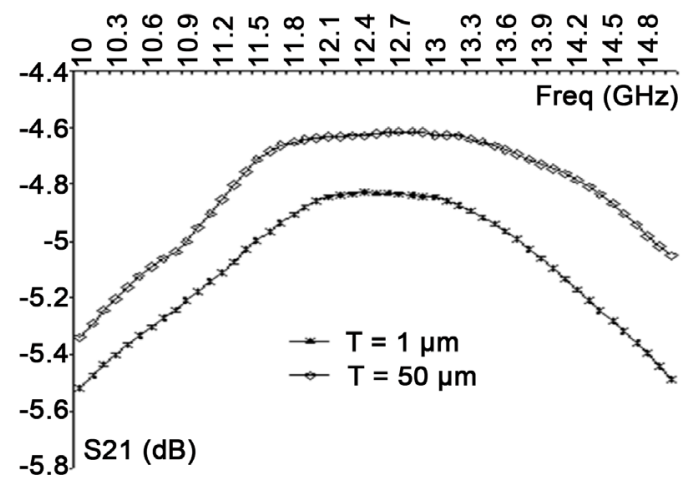

Figure 18. Variation of the transmission coefficient " $S_{21}$ " based on based on the thickness $T$ of the metal strip and the frequency. 
These curves show that if we increase the thickness of the metal strips the transmission between the two sources of excitations increases regardless of the operating frequency.

\section{Effect of Housing on the Results of the Problem}

We see in the results of 15 and 16 to a certain oscillation frequency 42.2 GHZ which is mainly due to the resonance of the housing. This oscillation is also observed in the results given by HFSS and ADS software. In fact to justify this we proceed to demonstrate.

We start with the ADS software. We compare the results of simulations of the structure with and without housing Figure 19 and Figure 20 shows the results when we see that this oscillation appears only in the results of the structure with the presence of the housing and is located $42.8 \mathrm{GHZ}$ frequency.

Freq $(\mathrm{GHz})$

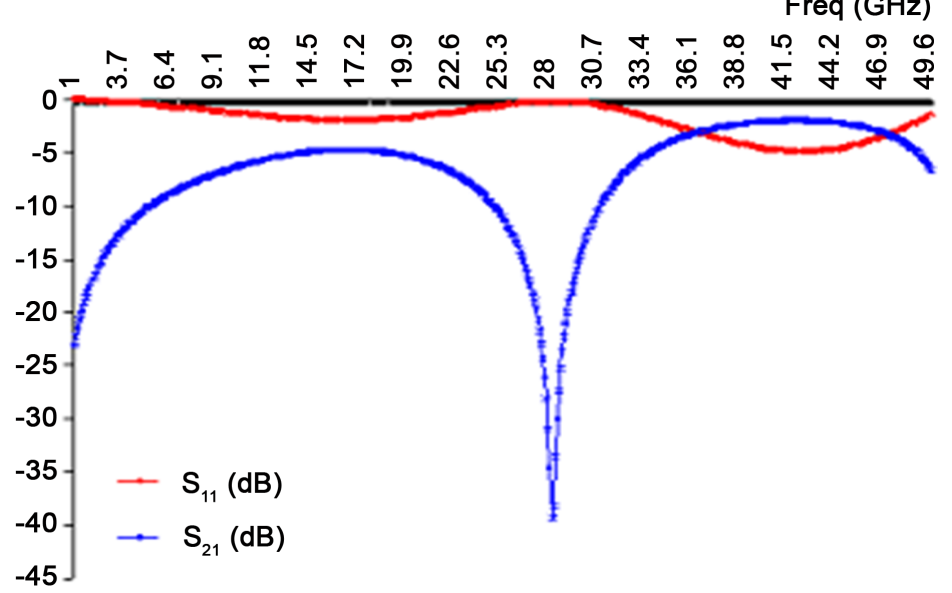

Figure 19. Coefficients of transmission and reflection versus frequency (without the presence of the housing).

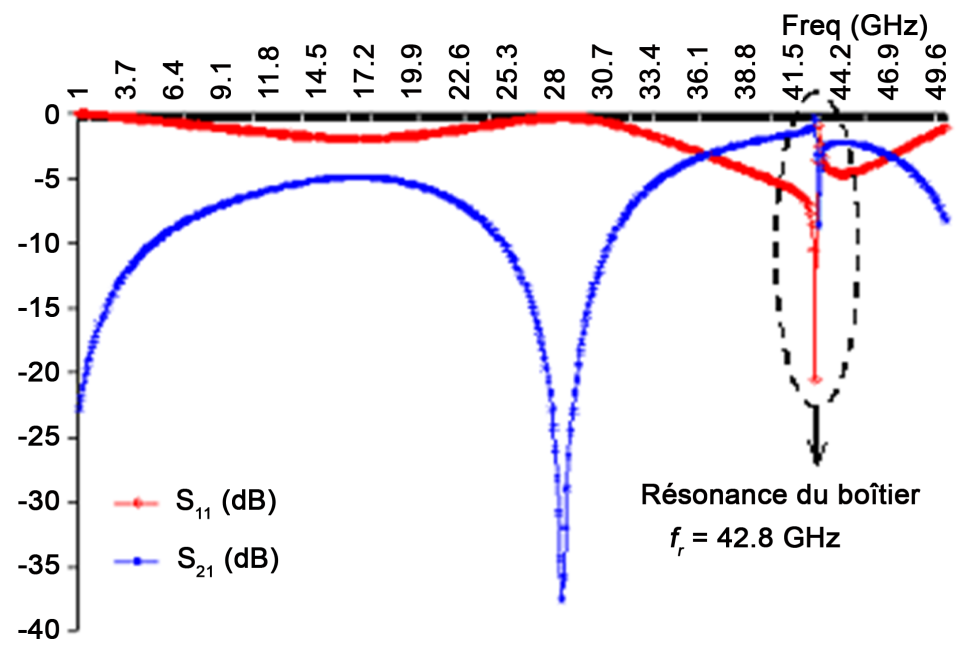

Figure 20. Coefficients of transmission and reflection versus frequency (with the presence of the housing). 
Figure 21 and Figure 22 show the resonance of the housing. They are derived from the difference of the results of the two curves of Figure 19 and Figure 20.

We are interested in our method of analysis. We take a metal housing walls that we excited by two sources of localized fields, but without the presence of micro-strip lines. We keep the same physical parameters of the study structure (Figure 23).

The different results obtained by simulation of the coefficients of reflection and transmission (Figure 24 and Figure 25), show the persistence of the resonance even in the absence of micro-strip lines, which confirms the presence of a resonance of the housing in the structure.

\section{Conclusion}

This article allowed us to review an electromagnetic model with what we have characterized as a planar structure including a flat, thick copper conductor. Indeed this model which is based on the phenomenon of skin effect encouraged us to model the latter two metal ribbons without thickness, placed one above the other which has a $h_{2}$ distance equal to the thickness $\mathrm{T}$ of conductor. Both sides, parallel to the plane Oyz, driver summers have been neglected because width of the metal is strictly greater than its thickness. This is a simplifying assumption which has no effect on the results of the

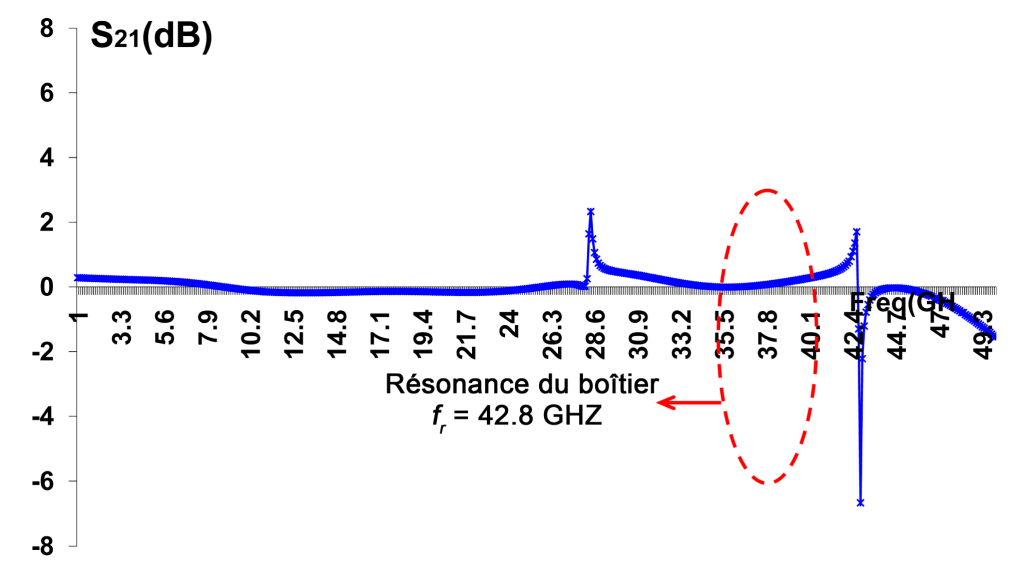

Figure 21. Resonance of the box.

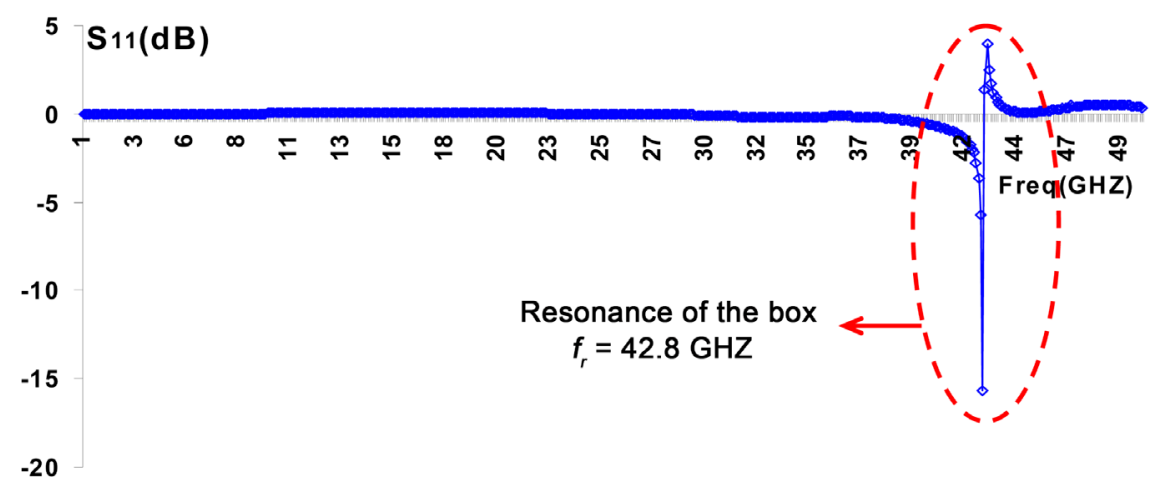

Figure 22. Resonance of the box. 


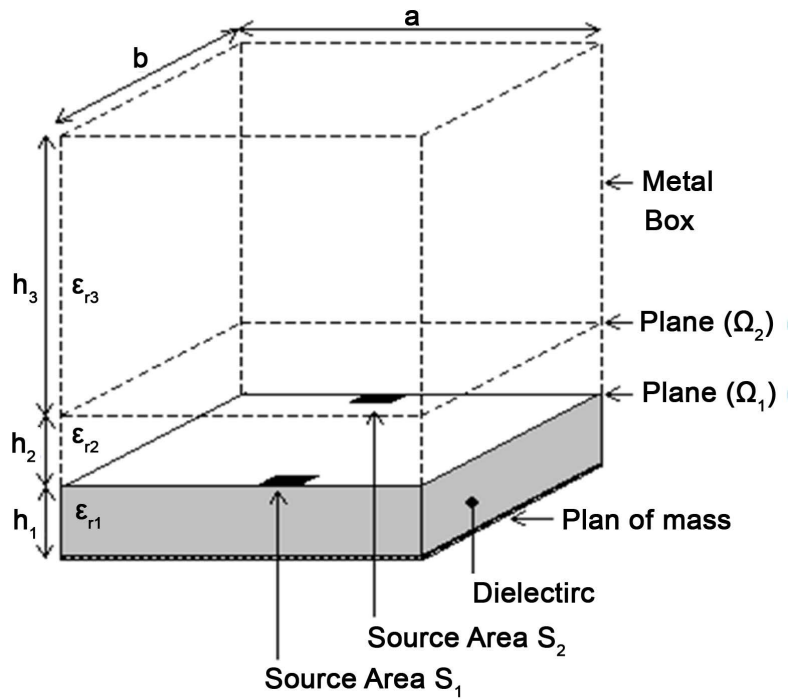

Figure 23. Excitement of the housing of the study structure by two planar and localized sources.

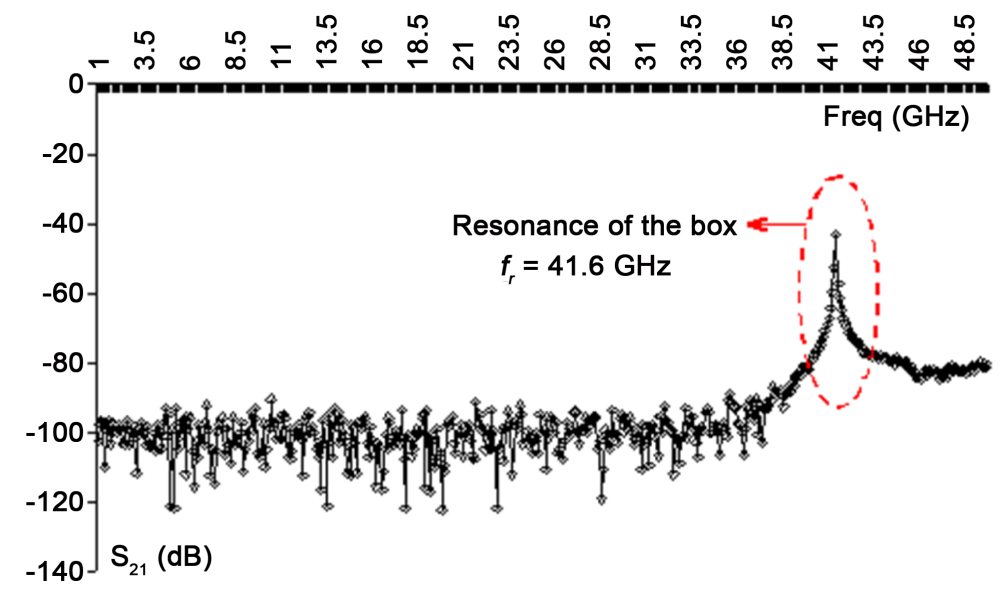

Figure 24. Resonance of the metal box.

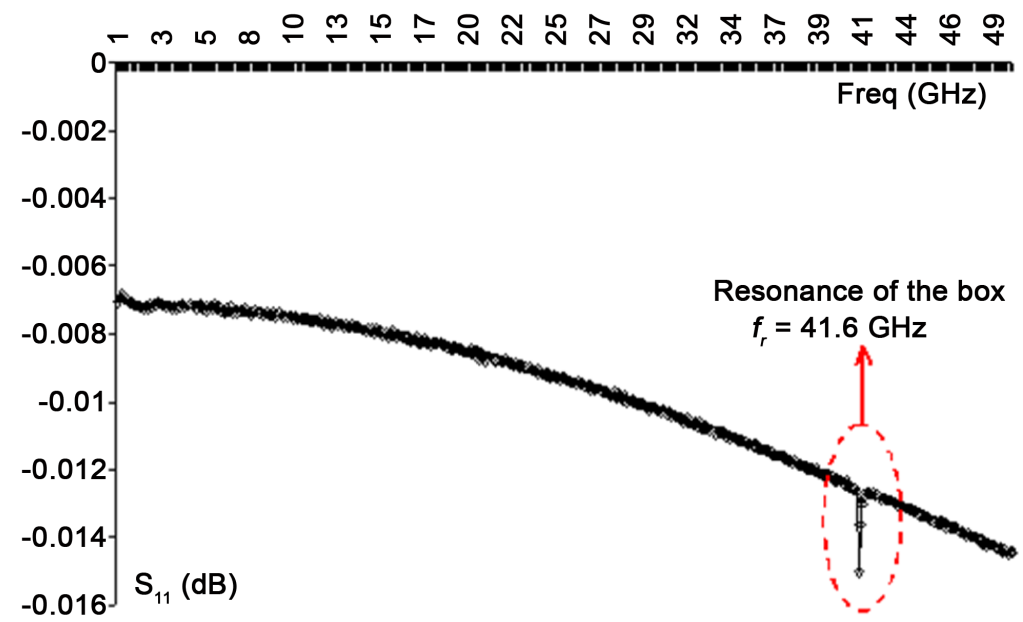

Figure 25. Resonance of the metal box. 
problem. The medium containing the thick conductor consists of a metal complex permittivity $\tilde{\varepsilon}^{\prime \prime}$ region (flat conductor thickness of copper T), and the rest of this medium is filled with air $\varepsilon_{r 2}=1$. The effective permittivity modeling the medium containing the driver is complex $\tilde{\varepsilon}_{\text {eff }}$. It is calculated from these two relative permittivity $\varepsilon_{r 2}$ and $\tilde{\varepsilon}^{\prime \prime}$. This approach has been implemented and tested by the multilayer iterative method (FWCIP). Simulations results found were compared with those calculated by the software Ansoft HFFS and ADS of Agilent Technology. They are in good matching, validating the method of analysis used.

\section{Acknowledgements}

This work has been supported by the SYSCOM laboratory, National Engineering School of Tunis Tunis El Manar University.

\section{References}

[1] Yeung, L.K. and Wu, K.-L. (2013) PEEC Modeling of Radiation Problems for Microstrip Structures. IEEE Transactions on Antennas and Propagation, 61, 3648-3655. http://dx.doi.org/10.1109/TAP.2013.2254691

[2] Wang, X.-H., Zhang, H.L. and Wang, B.-Z. (2013) A Novel Ultra-Wideband Differential Filter Based on Microstrip Line Structures. IEEE Microwave and Wireless Components Letters, 23, 128-130. http://dx.doi.org/10.1109/LMWC.2013.2243719

[3] Serres, A., Serres, G.K.F., Fontgalland, G., Freire, R.C.S. and Baudrand, H. (2014) Analysis of Multilayer Amplifier Structure by an Efficient Iterative Technique. IEEE Transactions on Magnetics, 50, Article Number: 7004404. http://dx.doi.org/10.1109/tmag.2013.2285601

[4] Jan, J.-Y., Pan, C.-Y., Chang, F.-P., Wu, G.-J. and Huang, C.-Y. (2012) Realization of Compact and Broadband Performances Using the Microstrip-Line-Fed Slot Antenna. 2012 Asia-Pacific Microwave Conference Proceedings (APMC), Taiwan, 4-7 December 2012, 1379-1381. http://dx.doi.org/10.1109/APMC.2012.6421925

[5] Kosslowski, S. (1988) The Application of the Point Matching Method to the Analysis of Microstrip Lines with Finite Metallization Thickness. IEEE Transactions on Microwave Theory and Techniques, 36, 1265-1271. http://dx.doi.org/10.1109/22.3668

[6] Feng, N.N., Fang, D.D. and Huang, W.P. (1998) An Approximate Analysis of Microstrip Lines with Finite Metallization Thickness and Conductivity by Method of Lines. Proceedings of the International Conference on Microwave and Millimeter Wave Technology, Beijing, 18-20 August 1998, 1053-1056. http://dx.doi.org/10.1109/ICMMT.1998.768471

[7] Farina, M. and Rozzi, T. (2000) Spectral Domain Approach to 2D-Modelling of Open Planar Structures with Thick Lossy Conductors. IEE Proceedings-Microwaves, Antennas and Propagation, 147, 321-324. http://dx.doi.org/10.1049/ip-map:20000732

[8] Shih, C. (1989) Frequency-Dependent Characteristics of Open Microstrip Lines with Finite Strip Thickness. IEEE Transactions on Microwave Theory and Techniques, 37, 793-795. http://dx.doi.org/10.1109/22.18856

[9] Henri, B., Sidima, W. and Damirnne, B. (2002) The Concept of Waves: Theory and Applications in Electronic Problems. Proceeding of the 9 th International Conference on Mathematical Methods in Electromagnetic Theory, Kiev, 10-13 September 2002, 100-104. http://dx.doi.org/10.1109/MMET.2002.1106841

[10] Serres, A., Fontgalland, G., de Farias, J.E.P. and Baudrand, H. (2010) An Efficient Algo- 
rithm for Planar Circuits Design. IEEE Transactions on Magnetics, 46, 3441-3444.

http://dx.doi.org/10.1109/TMAG.2010.2044150

[11] Kaddour, M., Mami, A., Gharsallah, A., Gharbi, A. and Baudrand, H. (2003) Analysis of Multilayer Microstrip Antennas by Using Iterative Method. Journal of Microwaves and Optoelectronics, 3, 39-52.

[12] Mejri, R. and Aguili, T. (2016) A New Approach Based on Iterative Method for the Characterization of a Micro-Strip Line with Thick Copper Conductor. Journal of Electromagnetic Analysis and Applications, 8, 95-108. http://dx.doi.org/10.4236/jemaa.2016.85010

Submit or recommend next manuscript to SCIRP and we will provide best service for you:

Accepting pre-submission inquiries through Email, Facebook, LinkedIn, Twitter, etc. A wide selection of journals (inclusive of 9 subjects, more than 200 journals)

Providing 24-hour high-quality service

User-friendly online submission system

Fair and swift peer-review system

Efficient typesetting and proofreading procedure

Display of the result of downloads and visits, as well as the number of cited articles Maximum dissemination of your research work

Submit your manuscript at: http://papersubmission.scirp.org/

Or contact jemaa@scirp.org 